\title{
Bacterial Recovery from Canine Eyes with Complicated Corneal Ulcerations: Comparison of Direct Plating Versus Culturette Submission: A Pilot Study
}

\author{
Haley E Jost ${ }^{1}$, Christine C Lim², Leslie Sharkey ${ }^{3}$, Julia L Sharp ${ }^{4}$, \\ Michael L Creutzinger ${ }^{4}$ and Michala de Linde Henriksen ${ }^{1 *}$ \\ ${ }^{1}$ Comparative Ophthalmology, Department of Clinical Sciences, College of \\ Veterinary Medicine and Biomedical Sciences, Colorado State University, USA \\ ${ }^{2}$ Eye Care for Animals, USA \\ ${ }^{3}$ Clinical Sciences Department, Cummings School of Veterinary Medicine, Tufts \\ University, USA \\ ${ }^{4}$ Department of Statistics, College of Natural Sciences, Colorado State University, \\ USA \\ *Corresponding Author: Michala de Linde Henriksen, Comparative Ophthalmology, \\ Department of Clinical Sciences, College of Veterinary Medicine and Biomedical \\ Sciences, Colorado State University, USA.
}

DOI: 10.31080/ASVS.2022.04.0322

\begin{abstract}
Objective: To compare organism recovery from canine corneal ulcers using two different bacterial culture processing methods: culturette inoculation into growth media immediately after collection (direct plating), and transport of culturettes with inoculation at the reference laboratory (culturette).

Design: Exploratory pilot study.

Animals: Thirteen client-owned dogs diagnosed with complicated corneal ulceration from one referral hospital.

Procedures: Bacterial samples were collected by direct sampling of infected corneal ulcerations with a culturette followed by two methods of sample processing: 1) direct inoculation of the culturette onto four culture media (blood, MacConkey, chocolate, Sabouraud dextrose) and shipment of plates to an outside laboratory, 2) sample collection by culturette followed by transport to the same outside laboratory for plating there for aerobic bacterial culture and sensitivity testing. Corneal cytology was collected from all corneal ulcerations immediately after culture samples were obtained.

Results: Direct plating detected bacterial infection in 5/13 (39\%) dogs, culturette submission in 6/13 (46\%) dogs. When combining the two culture methods, $7 / 13$ (54\%) dogs had positive cultures. The most common bacteria that were cultured from the corneal ulcerations were beta-hemolytic streptococcus spp. $(n=3)$, and gram-negative bacilli $(n=3)$. There was not sufficient evidence to suggest bacterial detection differed between the two culture methods $(\mathrm{p}=1.00)$.

Conclusions and Clinical Relevance: Some laboratories recommend direct plating for better bacterial growth from corneal cultures. Direct plating is more time consuming than submitting a culturette. This study suggests that submitting a culturette does not result in different bacterial growth than direct plating.
\end{abstract}

Keywords: Canine; Culturette; Direct Plating; Infected Corneal Ulceration; Microbiology

Citation: Haley E Jost, et al. "Bacterial Recovery from Canine Eyes with Complicated Corneal Ulcerations: Comparison of Direct Plating Versus Culturette Submission: A Pilot Study". Acta Scientific Veterinary Sciences 4.3 (2022): 03-10. 


\section{Introduction}

Bacterial ulcerative keratitis, also known as a type of complicated corneal ulceration, is one of the most common canine ocular diseases [1]. If not properly treated, this painful disease can progress rapidly due to both bacterial and host-derived factors (i.e., toxins and proteinases), possibly leading to loss of vision or even loss of the globe $[2,3]$. When a corneal ulceration is complicated by bacterial infection, medical management is lengthy, costly, and may eventually require surgical intervention to save the eye [1]. Treatment of complicated corneal ulcerations usually includes frequent application of topical antibiotics, anti-collagenolytics, mydriatic agents, and oral pain medications [1]. Successful resolution requires appropriate antimicrobial therapy ideally based on culture and sensitivity results $[1,4,5]$. However, due to the delay between culture submission and results, broad-spectrum antimicrobial selection is usually instituted prior to obtaining culture results [1]. Unfortunately, this contributes to poor antimicrobial stewardship that contribute to emerging resistance within populations of commonly isolated organisms and subsequent treatment failure. Common bacterial organisms isolated from complicated corneal ulcerations worldwide include Staphylococcus spp., Streptococcus spp., Pasteurella multocida, Pseudomonas aeruginosa, Corynebacterium spp., Neisseria spp., and Escherichia coli [4,6-9]. There have been many recent studies in veterinary medicine confirming antimicrobial resistance in these bacteria, especially for topical aminoglycosides and second-generation fluoroquinolones [6,7,9-11].

The conventional technique for obtaining corneal bacterial samples utilizes culturette collection (containing culture media) and transport to a microbiology laboratory where the sample will be plated [8,12-15]. Another less common culture technique utilizes immediate (direct) innoculation of the bacterial swab at the time of collection, with transport of the agar plates. In conjunction with corneal culture, cytology is one of the most commonly used and cost-effective laboratory procedures available to veterinary practitioners [8]. Cytology has several advantages as a complement to bacterial culture. Results are available rapidly to help guide antimicrobial selection, samples are assessed for concurrent inflammation and intracellular localization of organisms that can help distinguish pathogens from contaminants, and in some cases cytology can identify fastidious organisms that are not amenable to culture or can demonstrate organisms when there is compromise of the culture samples. Studies have been performed to support culture is more sensitive than cytology, however there is an advantage to using both methods for positive bacterial identification $[15,16]$. Other less common diagnostic methods for bacterial identification in both veterinary and human medicine include PCR, serology, and next-generation DNA sequencing [8,17-20].

University of Minnesota's Veterinary Medical Center uses an outside laboratory for microbiology diagnostics. This laboratory recommends direct plating on four different agar plates (blood, MacConkey, chocolate, Sabouraud dextrose) for all corneal microbiology cultures to increase the likelihood of bacterial growth. Direct plating can be more challenging and time consuming for the clinician [21,22]. In a nationwide survey in the United States, less than $50 \%$ of human medical practitioners had access to supplies necessary for direct plating [21]. To the author's knowledge, there are no studies in veterinary medicine comparing direct plating with culturette submission. If the superior culture method can be identified and implemented, antibiotic selection for veterinary patients will be more accurate, presumably resulting in a higher success rate for medical management of complicated corneal ulcerations and improving antimicrobial stewardship. The aim of this study is to determine whether there is a difference in positive culture rates between direct plating and culturette submission from complicated corneal ulcerations in dogs, and whether previous antibiotic use influences culture results. A secondary goal is to evaluate how the ancillary diagnostic technique of cytology compares with bacterial culture for the detection of infectious agents.

\section{Materials and Methods}

\section{Animals studied}

All study procedures were approved by the University of Minnesota Institutional Animal Care and Use Committee (Protocol \#1511-33205A). In this exploratory pilot study, client-owned dogs presenting to the ophthalmology service at University of Minnesota's Veterinary Medical Center were enrolled from December 2015 to November 2016. To be included in the study, client-owned dogs were required to have a complete ophthalmic examination performed by a veterinary ophthalmologist and a clinical diagnosis of "complicated corneal ulceration". Data collected from the study patients included age, breed, sex, and previous topical antimicrobials used prior to presentation. Superficial corneal ulcerations that were diagnosed as either simple (non-infected) or indolent by a veterinary ophthalmologist were excluded from the study. 


\section{Ophthalmic examination}

Complicated corneal ulcerations were diagnosed following a thorough ophthalmic examination performed by a board-certified veterinary ophthalmologist (CCL or MdLH). Examinations included Schirmer tear test (Merck Animal Health, USA), tonometry (Tonovet, icare, Finland $\mathrm{Oy}$ ), neuro-ophthalmic evaluation including menace response, dazzle reflex, pupillary light reflex (direct and indirect), and palpebral reflex, as well as slit-lamp biomicroscopy (Kowa Ltd, Japan) of adnexal, and anterior segment structures. Stromal loss and cellular infiltration of the corneal ulceration were identified on slit lamp biomicroscopy. Binocular indirect ophthalmoscopy (Keeler Inc., Malvern, PA, USA) with a 28D or 2.2D condensing lens (Volk Optical, Mentor, $\mathrm{OH}$ ) was performed on patients unless corneal opacity and/or miotic pupil precluded fundic examination. Culture and cytology samples were obtained per the study protocol (see 'Specimen collection' below). Following culture and cytology, fluorescein stain (Bio-Glo; HUB Pharmaceuticals, Scottsdale, AZ, USA) was applied to both eyes (OU).

\section{Specimen collection}

Prior to collection of corneal samples, one drop of topical anesthetic $(0.5 \%$ proparacaine hydrochloride, Akorn Inc, Lake Forest, Illinois, USA) was applied to the cornea. To maximize corneal anesthesia, a second drop was applied five minutes after the first application [23]. A new bottle of proparacaine was used for each new patient to prevent contamination. Two culture samples (one sample for direct plating and one sample for culturette submission) and three cytology samples were collected from each affected eye by CCL or MdLH. The order for which the two different cultures were collected was randomized based off a coin flip. For the direct plating method, four different agar platers were removed from the refrigerator 30 minutes prior to culture collection. A sterile culturette swab (COPAN, Murrieta, CA, USA) was used to gently rub the complicated corneal ulceration, using care to avoid contact with any adnexal tissues or hair. The swab was then directly plated on four different agar plates in the same order each time (blood, MacConkey, chocolate, Sabouraud dextrose). The plating was performed in the ophthalmology examination room immediately following sample collection. The plates were then submitted to the hospital laboratory for incubation until shipment to an outside laboratory (Marshfield Labs, Marshfield, WI). For the culturette submission method, the complicated corneal ulceration was sampled in the same fashion, but the culturette swab was then placed in media within a standard aerobic and anaerobic culturette tube (BBL $^{\mathrm{TM}}$ CultureSwab ${ }^{\mathrm{TM}}$ Plus, Copan Italia SpA, Italy). The culturette was shipped to the same outside laboratory as for the direct plating. The outside laboratory would perform the plating upon arrival on the same four agar plates as described above. Culture samples were stored at room temperature prior to shipping, and time from arrival to analysis was on average three days.

Cytology was collected from all patients as a supportive diagnostic test to microbiology, to evaluate for presence and type of inflammation and presence or absence of intracellular bacteria. Cytology samples were collected immediately after the culture sampling was complete. Three cytology slides were collected on each patient, using a new sterilized cytobrush (Microbrush, Grafton, WI) for each microscope slide. Cytology slides were submitted to the clinical pathology laboratory at University of Minnesota's Veterinary Medical Center. Two smears were stained with Wright Giemsa and one slide was stained with Gram stain. All cytologic preparations were evaluated by board-certified veterinary clinical pathologists.

\section{Data analysis}

The age, sex, and breed of each dog were recorded, and descriptive statistics were computed. Summary statistics (frequency and relative frequency) were computed for each culture and cytology result. Comparisons of interest were as follows: (i) culture results of direct plating versus culturette submission, (ii) detection of bacteria using microbiology culture versus detection of bacteria on cytology (bacteria +/- neutrophils), and (iii) positive culture rates between eyes that received previous antimicrobial therapy versus eyes that had not received antimicrobial therapy. To compare microbiology culture with cytology, the results of direct plating and culturette submission were combined by recording a 'presence' if at least one of the culture samples was positive for bacteria, and 'absence' if both cultures were negative for bacterial growth.

McNemar's Test, adapted for small sample sizes, was used to compare the yield of culture by means of direct plating versus culturette. All statistical analyses were carried out in the statistical software "R" (R Core Team (2020)). A significance level of 0.05 was used for all statistical tests.

\section{Results}

A total of 13 dogs were included in this study. All dogs were unilaterally affected with a complicated corneal ulceration. The median age was 10 years of age (range 6-15 years), including seven neutered males, five spayed females, and one intact female. Eight different dog breeds were represented in the study population, the 
Bacterial Recovery from Canine Eyes with Complicated Corneal Ulcerations: Comparison of Direct Plating Versus Culturette Submission: A Pilot Study

most common being the Boston Terrier $(n=4)$ followed by Boxer $(\mathrm{n}=2)$. The remaining breeds included Shih Tzu $(\mathrm{n}=1)$, Dachshund $(n=1)$, Miniature Pinscher $(n=1)$, Chihuahua $(n=1)$, Vizsla $(\mathrm{n}=1)$, and West Highland White Terrier $(\mathrm{n}=1)$. Eight out of 13 (62\%) complicated corneal ulcerations occurred in the right eye (OD), while five out of 13 (38\%) complicated corneal ulcerations occurred in the left eye (OS).

\section{Culture}

The direct plating method detected the presence of bacterial infection in five out of 13 complicated corneal ulcerations (39\%). The culturette submission method detected the presence of bacterial infection in six out of 13 complicated corneal ulcerations (46\%). A total of 11 out of 26 culture samples (42\%) had positive bacterial growth. When combining the two culture methods' results, seven out of 13 complicated corneal ulcerations (54\%) were positive for bacterial growth. Four out of five $(80 \%)$ eyes with a positive culture using the direct plating method also had a positive culture using the culturette collection method. Four of six (67\%) eyes with a positive culture using the culturette collection method also had a positive culture using the direct plating method. Both the direct plating and the culturette collection methods had identical culture results in ten out of 13 eyes (77\%; 4 eyes with positive cultures, 6 eyes with negative cultures). There was no evidence of a difference in culture yield between the two culture methods $(p=1.00)$. Culture results are summarized in table 1.

\begin{tabular}{|l|c|c|c|c|c|}
\hline \multicolumn{2}{|c|}{ Bacteria Detection Method } & \multicolumn{2}{c|}{ N } & \multicolumn{1}{c|}{ Odds Ratio (95\% CI) } & P \\
\hline Direct plating & & 5 & $(38.5)$ & & \\
\hline Culturette & & 6 & $(46.2)$ & & 1.00 \\
\hline Detection agreement & & 10 & $(76.9)$ & $2.00(0.104,117.994) *$ & \\
\hline & Presence & 4 & $(30.8)$ & & \\
\hline & Absence & 6 & $(46.2)$ & & \\
\hline Cultures (combined) & & 7 & $(53.8)$ & & .375 \\
\hline Cytology (bacteria) & & 4 & $(30.8)$ & & \\
\hline Detection agreement & & 8 & $(61.5)$ & $0.25(0.005,2.526)$ & \\
\hline & Presence & 3 & $(23.1)$ & & 1.00 \\
\hline & Absence & 5 & $(38.5)$ & & \\
\hline Cultures (combined) & & 7 & $(53.8)$ & & \\
\hline Cytology (neutrophils) & & 8 & $(61.5)$ & & \\
\hline Detection agreement & & 6 & $(46.2)$ & $1.33(0.226,9.102)$ & \\
\hline & Presence & 4 & $(30.8)$ & & \\
\hline & Absence & 2 & $(15.4)$ & & \\
\hline Cytology (bacteria) & & 4 & $(30.8)$ & & \\
\hline Cytology (neutrophils) & & 8 & $(61.5)$ & & \\
\hline Detection agreement & & 9 & $(69.2)$ & & \\
\hline & Presence & 4 & $(30.8)$ & & \\
\hline & Absence & 5 & $(38.5)$ & & \\
\hline
\end{tabular}

Table 1: Summary statistics (frequency (\%)) for each comparisons (direct plating vs culturette; cultures (combined) vs cytology (intracellular bacteria); cultures (combined) vs cytology (neutrophils), cytology (intracellular bacteria) vs. cytology (neutrophils)); ' $n$ ' in the second column indicates the number of dogs with positive bacterial growth on their culture method, and '\%' indicates the frequency of when the methods agreed on presence and absence $(n=13) .{ }^{*}$ Odds ratio can be interpreted as the odds of detection with culturette is approximately twice the odds of detection with direct plating. ${ }^{\bullet}$ Odds ratio estimate not calculated due to having zero observations that were detected as positive by cytology (bacteria), and negative by cytology (neutrophils). 
Eight different bacterial isolates were identified between the two culture methods: Beta-hemolytic Streptococcus spp. $(35 \%, \mathrm{n}=$ $6)$, gram-negative bacilli $(12 \%, \mathrm{n}=2)$, Staphylococcus intermedius $(12 \%, \mathrm{n}=2)$, Escherichia coli $(12 \%, \mathrm{n}=2)$, Corynebacterium ulcerans $(12 \%, \mathrm{n}=2)$, Staphylococcus aureus $(6 \%, \mathrm{n}=1)$, Capnocytophagia spp. $(6 \%, \mathrm{n}=1)$, Enterobacter cloacae $(6 \%, \mathrm{n}=1)$. Six of 11 eyes (55\%) yielded one isolate, four of 11 eyes (36\%) yielded two isolates, and one of 11 eyes ( $9 \%$ ) yielded three isolates. No sample yielded more than three isolates.

\section{Cytology}

Eight of 13 (62\%) cytology results from the complicated corneal ulcerations showed evidence of suppurative inflammation with no etiologic agent identified ("neutrophils only"). Of those eight eyes, four (50\%) were positive for bacteria on aerobic culture. Four out of 13 (31\%) eyes identified intracellular bacteria on cytology ("bacteria +/- neutrophils"). Of those four eyes, three (75\%) also had a positive bacterial culture, and one (25\%) was negative on both culture methods. One hundred percent of eyes positive for intracellular bacteria on cytology also showed evidence of suppurative inflammation. Additionally, four out of 13 (31\%) eyes that had a positive bacterial culture (using either culture method) but were negative for bacteria on cytology. When combining the culture results of direct plating with culturette submission, aerobic culture detected the presence of bacteria $(54 \%, 7 / 13)$ more frequently than cytology $(31 \%, 4 / 13)$, however there was not sufficient evidence to suggest a difference between culture and cytology detection $(\mathrm{p}=.375)$.

\section{Antibiotic use}

Of the 13 dogs in the study, seven of 13 complicated corneal ulcerations (54\%) were on a topical antibiotic at the time of culture and cytology collection. The most common topical antibiotic was neomycin-polymyxin B-bacitracin ophthalmic ointment (Bausch \& Lomb Incorporated, Tampa, FL, USA; $n=3$ ), followed by tobramycin $0.3 \%$ ophthalmic solution (Bausch \& Lomb, Bridegwater, NJ, USA; $n$ = 2), ofloxacin $0.3 \%$ ophthalmic solution (Akorn, Inc., Lake Forest, IL, USA; $n=1$ ) and ciprofloxacin $0.3 \%$ ophthalmic solution (Altaire Pharmaceuticals, Aquebogue, NY, USA; $n=1$ ). Four of seven complicated corneal ulcerations (57\%) that were treated with a topical antibiotic were negative for bacterial growth on their microbiology culture, while three of seven complicated corneal ulcerations
(43\%) that were treated with a topical antibiotic were positive for bacterial growth. Of the six complicated corneal ulcerations without topical antibiotic use at the time of specimen collection, two out of six (33\%) was negative for bacterial growth, and four out of six $(67 \%)$ were positive for bacterial growth. There was not sufficient evidence to suggest a difference between bacterial growth or no growth when the use of topical antibiotics versus no use of topical antibiotics prior to culture were evaluated $(\mathrm{p}=.85)$. The use of systemic antibiotic therapy was not recorded for any of the 13 dogs in this study.

\section{Discussion}

This exploratory pilot study compared culture results of complicated corneal ulcerations from direct plating and culturette submission. The direct plating method was positive for bacterial culture in $39 \%$ of cases, with culturette submission positive in $46 \%$ of cases, though this difference was not found to be statistically significant. A similar study was performed in human ophthalmology to determine the microbiological yield of corneal ulceration by direct inoculation of culture media versus indirect inoculation by means of transport medium [24]. Like the current study, Mcleod., et al. 2005 reported that cultures obtained by means of culturette submission and held for up to 24 hours had similar positive culture results to that of direct plating. The results of this study suggest that direct plating and culturette submission are equivalent for obtaining positive culture results. Given added time and technical demands of direct plating, culturette transport appears to be the more practical alternative for most practitioners.

It is important to note that while swab transport devices are used in veterinary and human ophthalmology for ease of sample collection, swab specimens are inferior to fluid specimens (i.e., wound exudate), thus culture yield may be lower in these cases [25]. Along with potential for decreased positive culture yields with complicated corneal ulcerations, topical antibiotic use prior to diagnostics must be considered as a confounding variable regarding culture results. A 2007 study by Sharma and colleagues evaluated positive and negative culture results correlated with cytology and prior antibiotic use. The study found that more negative cultures were associated with previous topical antibiotic therapy, however this was not statistically significant [26]. A similar observation in the present study was demonstrated, as there was no evidence to suggest a difference between culture rates of eyes on prior topi- 
cal antibiotic use with eyes that had not received a topical antibiotic prior to culture collection. Additionally, one must consider the low culture rate of fastidious organisms related to negative culture results. In a 2008 study by Ledbetter and colleagues, mixed infections with aerobic bacteria were present in $51.2 \%$ of cases when attempting to isolate obligate anaerobes. The authors suspected this was an under-estimation, as $76.2 \%$ of anerobic bacterial ulcers that were negative for aerobes were receiving topical antimicrobial therapy at presentation [27]. This may have been the case in the current study, as $57 \%$ of complicated corneal ulcerations that were previously treated with a topical antimicrobial were culture negative.

The commonly identified bacterial isolates in this study were beta-hemolytic Streptococcus spp., Staphylococcus spp., and gramnegative bacilli. This is similar to a recent study by Jinks., et al. from 2020, which identified Staphylococcus, Streptococcus, and Pseudomonas as the most common bacterial genera isolated in dogs [11]. Similar results have been demonstrated in other veterinary ophthalmology studies evaluating microbiology from dogs, cats, and horses [4,9].

In the current study, there was not enough evidence to suggest a difference between cytology or aerobic culture regarding positive bacterial identification, though combined culture methods detected bacteria in $54 \%$ of cases compared to $31 \%$ of cytology samples. Bacterial culture is known to be more sensitive for the identification of infectious agents, however cytology is still useful due to the ability to quickly obtain results [1]. Corneal cytology has also been previously reported to correlate with corneal culture results with a positive and negative predictive value (PPV and NPV) of $73 \%$ and $52 \%$, respectively, in the horse [28]. Another study showed the benefit of using more than one diagnostic technique to identify bacteria. In 48 horses with ulcerative keratitis, bacteria was identified on cytology in 26 out of 35 infections compared to 29 identified on bacterial culture, compared to 35 infections using combined methods [16]. Some discrepancies that lead to misdiagnosis from cytology can result from sampling technique. Additionally, certain normal findings such as stain precipitate can be mistaken for bacteria [8]. However, a preliminary diagnosis based on cytology can be crucial in early bacterial identification and implementation of appropriate antimicrobial therapy.

As the field of medicine continues to grow, advancements in microbial identification are becoming more promising. In humans, the limitations of culture methods for microbial identification were further elucidated by Willcox., et al. who showed that less than $80 \%$ of healthy human conjunctival swabs yielded cultivable microbes, despite a known endogenous conjunctival microbiome [29]. In another human study, next generation sequencing, using $16 \mathrm{~S}$ ribosomal DNA, was able to positively identify a highly diverse microbial conjunctival community in each of its samples, with $31 \%$ of microbes classified as "novel" bacteria [30]. In animals, nextgeneration sequencing has been used to characterize the ocular microbiota in the house finch, koala, feline, canine, and equine [31-35]. Most recently, a 2020 study by LaFrentz., et al. characterized the diversity of the equine conjunctival microbiota using nextgeneration DNA sequencing in 15 eyes [35]. Conjunctival biopsies yielded 329 bacterial operational taxonomic units (OTUs) and found gram negative Proteobacteria to be the dominant phylum. A similar study was performed in 2019 by Leis at al. to characterize the canine ocular microbiota and found that genera previously thought to account for the majority of the core microbiome only accounted for $2.63 \%$ of all sequencing reads, possibly reflecting bias in organism recovery using standard culture methods [34]. Further studies are warranted to characterize the general ocular microbiome among various species, but eventually, its use could lead to the development of novel therapies to prevent corneal dysbiosis or to identify uncommon fastidious bacterial isolates responsible for many complicated corneal ulcerations with negative culture results. This would prompt more judicious use of topical antibiotics to reduce the spread of antimicrobial-resistant organisms.

Based on results in this pilot study, direct plating and transport media had comparable positive culture rates. Of the positive cultures using both methods, nearly all isolates identified were identical. Given that direct plating on culture media is inherently more difficult and time-consuming, inoculation into transport medium is a viable and effective alternative. Additionally, as bacterial identification between cytology and culture methods did not significantly differ, implementing corneal cytology is an important way to quickly identify etiologic agents responsible for complicated corneal ulcerations, thus implementing appropriate treatment prior to obtaining culture results.

\section{Acknowledgment}

This study was funded by University of Minnesota's Frances LeClaire Ophthalmology grant. The authors would like to acknowledge Melissa Boyd, Stacy Bokelheide, Perrin Bullers-Schwartz, and 
Emily Metz Quandt at University of Minnesota Veterinary Medical Center for their help with this clinical study. The authors of this manuscript have no conflict of interest to declare.

The abstract of this manuscript has been presented at the 2021 American College of Veterinary Ophthalmologists (ACVO) Conference in Indianapolis, Indiana.

Bibliography

1. Gelatt KN., et al. "Ulcerative keratitis. IN: Gelatt KN, ed. Veterinary Ophthalmology". 6th ed. John Wiley and Sons, Ames, IA, USA (2021): 1096-1123.

2. Wang L., et al. "Evaluation of matrix metalloproteinase concentrations in precorneal tear film from dogs with Pseudomonas aeruginosa-associated keratitis". American Journal of Veterinary Research 69.10 (2008): 1341-1345.

3. Ollivier FJ., et al. "Proteinases of the cornea and preocular tear film". Veterinary Ophthalmology 10.4 (2007): 199-206.

4. Tolar EL., et al. "Evaluation of clinical characteristics and bacterial isolates in dogs with bacterial keratitis: 97 cases (19932003)". Journal of the American Veterinary Medical Association 228.1 (2006): 80-85.

5. Papich MG. "Antimicrobials, susceptibility testing, and minimum inhibitory concentrations (MIC) in veterinary infection treatment". Veterinary Clinics of North America: Small Animal Practice 43.5 (2013): 1079-1089.

6. Lin CT and Petersen-Jones SM. "Antibiotic susceptibility of bacterial isolates from corneal ulcers of dogs in Taiwan". Journal of Small Animal Practice 48.5 (2007): 271-274.

7. Hindley KE., et al. "Bacterial isolates, antimicrobial susceptibility, and clinical characteristics of bacterial keratitis in dogs presenting to referral practice in Australia". Veterinary Ophthalmology 19.5 (2016): 418-426.

8. Gould D., et al. "Ocular bacteriology. In: Gelatt KN, ed. Veterinary Ophthalmology. $6^{\text {th }}$ ed. John Wiley and Sons, Ames, IA, USA (2021): 308-319.

9. Suter A., et al. "Septic keratitis in dogs, cats, and horses in Switzerland: associated bacteria and antibiotic susceptibility". Veterinary Ophthalmology 21.1 (2018): 66-75.

10. Sandmeyer LS., et al. "Alterations in conjunctival bacteria and antimicrobial susceptibility during topical administration of ofloxacin after cataract surgery in dogs". American Journal of Veterinary Research 78.2 (2017): 207-214.
11. Jinks MR., et al. "Using minimum inhibitory concentration values of common topical antibiotics to investigate emerging antibiotic resistance: A retrospective study of 134 dogs and 20 horses with ulcerative keratitis". Veterinary Ophthalmology 23.5 (2020): 806-813.

12. Whitley DR. "Canine and Feline Primary Ocular Bacterial Infections". Veterinary Clinics of North America: Small Animal Practice 30.5 (2000): 1151-1167.

13. Furiani N., et al. "Evaluation of the bacterial microflora of the conjunctival sac of healthy dogs and dogs with atopic dermatitis". Veterinary Dermatology 22.6 (2011): 490-496.

14. Ollivier FJ. "Bacterial corneal diseases in dogs and cats". Clinical Techniques in Small Animal Practice 2003;18 (3): 193-198.

15. Galán A., et al. "Clinical findings and progression of 10 cases of equine ulcerative keratomycosis (2004-2007)". Equine Veterinary Education 21.5 (2009): 236-242.

16. Massa KL., et al. "Usefulness of aerobic microbial culture and cytologic evaluation of corneal specimens in the diagnosis of infectious ulcerative keratitis in animals". Journal of the American Veterinary Medical Association 215.11 (1999): 1671-1674.

17. Banks KC., et al. "Veterinary ocular microbiome: Lessons learned beyond the culture". Veterinary Ophthalmology 22.5 (2019): 716-725.

18. Martín R., et al. "The role of metagenomics in understanding the human microbiome in health and disease". Virulence 5.3 (2014): 413-423.

19. Morgan $\mathrm{XC}$ and Huttenhower C. "Human microbiome analysis". PLOS Computational Biology 8.12 (2012): e1002808.

20. Borroni D., et al. "Metagenomics in ophthalmology: current findings and future prospectives". BMJ Open Ophthalmology 4.1 (2019): e000248-e000248.

21. McLeod SD., et al. "Differential care of corneal ulcers in the community based on apparent severity". Ophthalmology 103.3 (1996): 479-484.

22. McDonnell PJ. "Empirical or culture-guided therapy for microbial keratitis?: A plea for data”. Archives of Ophthalmology 114.1 (1996): 84-87.

23. Binder DR and Herring IP. "Duration of corneal anesthesia following topical administration of $0.5 \%$ proparacaine hydrochloride solution in clinically normal cats". American Journal of Veterinary Research 67.10 (2006): 1780-1782. 
24. McLeod SD., et al. "Reliability of Transport Medium in the Laboratory Evaluation of Corneal Ulcers". American Journal of Ophthalmology 140.6 (2005): 1027-1031.

25. Perry JL. "Assessment of swab transport systems for aerobic and anaerobic organism recovery". Journal of Clinical Microbiology 35.5 (1997): 1269-1271.

26. Sharma S., et al. "Comparison of clinical and microbiological profiles in smear-positive and smear-negative cases of suspected microbial keratitis". Indian Journal of Ophthalmology 55.1 (2007): 21-25.

27. Ledbetter EC and Scarlett JM. "Isolation of obligate anaerobic bacteria from ulcerative keratitis in domestic animals". Veterinary Ophthalmology 11.2 (2008): 114-122.

28. Jeffery U., et al. "Ability of corneal cytology to predict bacterial culture results". Veterinary Clinical Pathology 41.4 (2012): E49.

29. Willcox MDP. "Characterization of the normal microbiota of the ocular surface". Experimental Eye Research 013.117 (2013): 99-105.

30. Dong Q., et al. "Diversity of Bacteria at Healthy Human Conjunctiva". Investigative Ophthalmology and Visual Science 52.8 (2011): 5408-5413.

31. Thomason CA., et al. "Eye of the Finch: characterization of the ocular microbiome of house finches in relation to mycoplasmal conjunctivitis". Environmental Microbiology 19.4 (2017): 1439-1449.

32. Alfano N., et al. "Variation in koala microbiomes within and between individuals: effect of body region and captivity status". Scientific Reports 5.1 (2015): 10189.

33. Weese SJ., et al. "The oral and conjunctival microbiotas in cats with and without feline immunodeficiency virus infection". Veterinary Research 46.1 (2015): 21.

34. Leis ML and Costa MO. "Initial description of the core ocular surface microbiome in dogs: Bacterial community diversity and composition in a defined canine population". Veterinary Ophthalmology 22.3 (2019): 337-344.

35. LaFrentz S., et al. "Characterization of the normal equine conjunctival bacterial community using culture-independent methods". Veterinary Ophthalmology 23.3 (2020): 480-488.

\section{Assets from publication with us}

- Prompt Acknowledgement after receiving the article

- Thorough Double blinded peer review

- Rapid Publication

- Issue of Publication Certificate

- High visibility of your Published work

Website: www.actascientific.com/

Submit Article: www.actascientific.com/submission.php

Email us: editor@actascientific.com

Contact us: +919182824667 